\title{
Thoughts on English Vocabulary Teaching based on Cognitive Linguistics
}

\author{
Lian Xue \\ School of Foreign Languages and Literature, Wuhan Donghu University, Wuhan Hubei, 430212, \\ China
}

Key words: Cognitive Linguistics, English Vocabulary, Teaching.

\begin{abstract}
For the overall Chinese education, impact brought by quality-oriented education is indeed an essential education concept innovation. For English learning, teachers should not only improve students' cognition on the knowledge structure, but also should guarantee the effective improvement of students' overall learning literacy to promote students form good English-learning habits and learning temperament. In the practical English teaching, cognitive linguistics theories are effective measures that ensure the further internalization of English vocabularies. Based on the fundamental philosophy of cognitive linguistics, this paper made a detailed analysis on the understanding of corresponding vocabulary and the knowledge structure, aiming to preferably improve teachers' inspiration on English vocabulary teaching.
\end{abstract}

\section{Introduction}

As an instrumental language discipline, in the process of practical teaching, English will involve a lot of problems relating to the construction of language patterns, when new language cognitive structure generates, the corresponding influence will come out, thus requires English teachings to timely update the corresponding way of language teaching in the process of practical teaching to ensure the timely upgrading of teaching concepts.

\section{Teaching of polysemous words in cognitive linguistics}

In Chinese learning, we often meet vocabulary and word that has several meanings, normally we understand it under the special situation, while the research and de-structure of language has a certain of universal property. There are also polysemous words in English, one situation is that the vocabulary itself has different meanings, it has different meanings in specific use.For example, pick up, this phrase is normally translated as "pick up", but under the special situation, it has another meaning "carry passenger on the midway", which needs teachers' specific instruction under the specific situation. Another situation is that words with the same shape but has different meanings, for example, as a verb, bear means "endure", as a noun, it means "bear"; as a modal verb, can means "be able to", but as a noun, it means "tin" and so on. In the traditional language analysis and research, research of polysemous words is just simple induction and aggregation, no complete basic knowledge structure.In the practical teaching, teachers also just make corresponding explanation for corresponding meanings, ignoring its culture background, no further research on the language prototype and presentation. Under this research background, the traditional classical category theory separates the research method in essence.Related language researchers should establish research mode for prototype-based category to ensure corresponding prototype form basic structure summary, make comprehensive research on the prototype radiation related meaning to ensure the completeness 
of whole English vocabulary meanings. Next, the author will make a concentrated analysis on the common word "about".

(1) My mother often spoke to me about you.

(2) She sat up and looked about her.

(3) I had no money about me.

(4)It happened about 5 o' clock in the morning.

(5) What the hell are you about?

(6)What I like about him is his sense of humor.

Explain "about” based on the cognitive linguistics philosophy, in English, it has meanings as preposition, adjective and adverb, mainly translated as "approximately, concerning", among which (1) (5) (6) are extended meaning of "concerning”, including "be engaged in; among”, while (2)(3)(4) are extended meaning of "approximately", including "around; by the side of”, extend from the original meaning, forming the overall meaning structure of the vocabulary. Therefore, in the specific use of language, it will form a structural language output way, it is easy to find that in the vocabulary or sentence, "about" can be used diversely, which provides a huge space for teachers' choice of teaching ways. In the practical teaching, it requires teacher to explain from a new perspective, integrate corresponding content to construct concentrated vocabulary knowledge. In the teaching, teachers can use the pattern of minding mapping to establish the theory structure and summary of polysemous words $^{[1]}$.

\section{Teaching of categorized vocabularies in cognitive linguistics}

In the research process of cognitive linguistics, research of vocabulary category and categorization are basic research key points, are the cognitive induction of English knowledge. In our real life, everything has a certain category value, all are explained within a certain scope, we make concentrated category and analysis on corresponding events thus to establish correct cognition for the world. For example, we make basic category for animals in English, (1)Vertebrate and invertebrate (2) Viviparous animal and ovipara animal (3)Metazoan and unicellular animal, these is macrotaxonomy for animals which forms the basic animal category, there are no influence on the real layers, in the practical teaching process, teachers' explanation for corresponding vocabularies is also a summary for the basic category. Analyze from the psychology angle, early childhood is the basic establishing period for category, children make initial category for basic things, and summarize by themselves. In the practical teaching process, teachers also need to make concentrated category for the application of English category. In the daily teaching, category has a huge boosting function for students' vocabulary cognition, students learn the vocabulary meanings and probably internalize and apply corresponding vocabularies through vocabulary categorization. Teachers should fully apply the research of categorization theory in the cognitive linguistics, ensure it is better than the basic teaching design to help students build the categorized knowledge.

There are many teaching instruments and medias to assist teaching, in the practical teaching process, teachers should make basic category for students' vocabulary, teachings' teaching time is limited, but the extended power of teaching method is limitless. Teachers help students to integrate English vocabularies within a corresponding category by using the category concept of cognitive linguistics to help them learn English vocabularies. If the bulk deposition of vocabulary has no real effect, it is just the change of amount but not the qualitative leap. Though students grasp some difficult vocabularies, they cannot apply them in the real communication and writing, because students have no categorized cognition of vocabularies. Teachers should encourage students to build their own vocabulary application category, when facing the real problem, they can fully mobilize their vocabulary using consciousness and form the corresponding behavior mode ${ }^{[2]}$.

\section{Teaching of meaningful vocabularies in cognitive linguistics}


In the traditional vocabulary teaching, the whole teaching concept makes an one-side type division for vocabularies and their real meaning, it thinks that the image and meaning of vocabularies has no necessary link, it is just the accumulation of independent letters. As shown in figure 1:
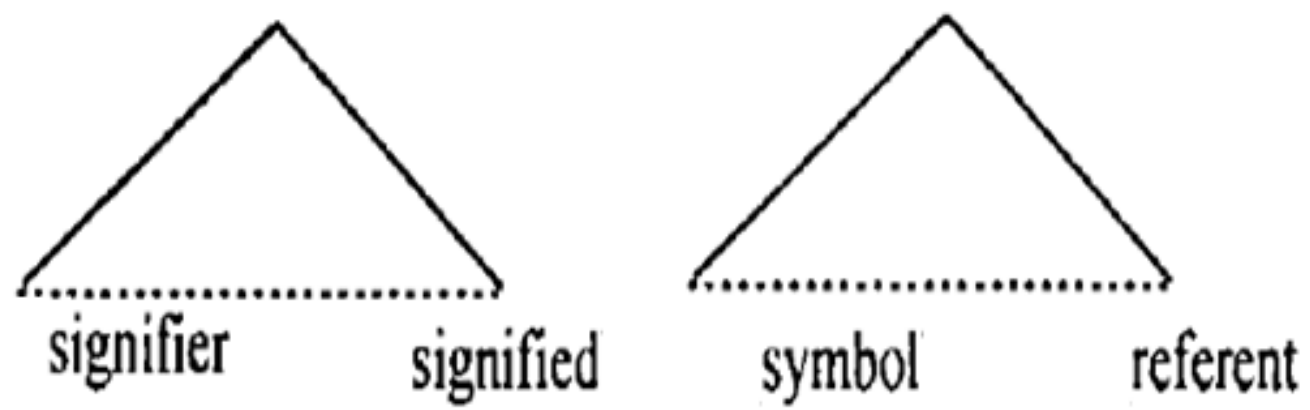

Fig. 1. The former is symbol, the latter is concept--the dotted lines represents that there are no internal connection of the two.

While in the cognitive linguistics, there is a rather rational upgrading of the corresponding theory, it thinks that in the process of English vocabulary design, the basic foundation of language meaning is based on cognitive basis. People often start from their own behavior mode to establish basic cognition for surrounding things thus to extend corresponding concept of time and space and make a separation for corresponding things based on their property. People's basic cognition of outside things come from accumulation of real experience, from the primitive to progress, from basic to architecture, from specific to abstract. During the specific interpretation process, teachers should establish vocabulary language model, establish English conceptual learning model for students, because vocabulary knowledge has corresponding English culture background, it can not only stimulate students' learning interest, but also can further stimulate students and teachers to form benign interaction, to better coordinate teachers' teaching behavior. When teacher explain the modal verbs, students' understanding on modal verb is always a difficult teaching problem, students cannot understand the specific context of corresponding vocabularies, they cannot establish complete cognition on the a series of modal verbs, like may/could/can/should/need/must/have to and so on. If teachers just always emphasize the usage of corresponding vocabularies, there will not have good teaching effects. Teachers should make emotion projection for corresponding language situation, establish the necessary connection between vocabularies and meanings, memorize with rational thinking, feel with emotional thinking to form good language structure thus can help students memorize vocabularies and real application. For example, the biggest distinction of have to and must is the difference of subjective moods and objective moods, teachers should make basic analysis for vocabularies during the teaching process. Another example, may is a basic figure between the material world and human society, it displays the smallest expression approach of modalization. When students internalize the modal verbs, the first information they received is the meaning and culture explained by the teacher, thus they can further understand the specific application, teachers should ensure students' basic cognition of teaching process, thus can provide convinced evidence for the overall teaching content ${ }^{[3]}$.

Therefore, it is very important for teachers to explain the meaning and value of vocabulary, to establish complete structure for the essential connotation of corresponding vocabulary, to encourage students form the learning model of active thinking, to emphasize the application of foreign language vocabulary in the real life, emphasize the further innovative consciousness and ability. If teachers want to achieve better effect in the lessons, they should change the teaching thinking ways in essence, make bold attempt on the ways and methods to ensure students get corresponding improvement ${ }^{[4]}$.

\section{Teaching of implicit vocabularies in cognitive linguistics}


In the traditional teaching pattern, when explain vocabularies, teachers should pay more attention to the vocabulary meaning in the unit and article, but not have extension for corresponding extra-curricular knowledge, have no detailed research on the implicit meaning of vocabularies. Students cannot form sufficient cognition on the overall concept, also will restrict students' operation in real application. While in the field of cognitive linguistics research, the implication connotation of vocabulary needs detailed discussion, point out in the specific theory, the metaphorical thoughts is established after the development of people's basic cognition, is an innovative understanding and construction of corresponding things, is a more sophisticated learning pattern. During the process that human being know the world, only ensuring the deep analysis and cognition of implicit knowledge can construct complete thinking and ensuring the improvement of knowledge cognition ability. Human being's understanding of the world fist originates from self cognition, then further de-structure the world through their own cognition concept. And during this process, human being will find out the basic essence of things through all kinds of images. With more and more cognition, people will ignore some problems in the adding and organization process of the overall analysis. Therefore, people start to research a new construction way, that is to realize relevance development and establishment by using metaphor. In the cognitive linguistics, metaphor is the basic thinking of human beings, it not only needs refer to the corresponding situation, but also needs to construct the thinking way for specific and abstract concept. For example, English word heart, in the real application, it means "heart", while with the extension of basic meanings, in many phrases, heart begin to have other meanings, in the heart of (in the center of)/take heart (have courage)/at heart(in essence)/my heart goes on/ cross my heart (I swear), the meaning of heart already has many implicit extension, not only just means the part of human body, related emotions also can use heart to express. The vocabulary implicit can also be footstone to improve students' interest, teachers can fully excavate students' curiosity to form a pointed characteristic teaching ${ }^{[5]}$.

\section{Conclusion}

All in all, English vocabulary learning is a multifarious big project for students, but also the basic guarantee for students to learn English well. Students not only need to recite basic English vocabulary, more need to use in the real communication. Teachers should optimize relevant content in the real class, fully practice relevant concept of cognitive linguistics, positively develop new teaching pattern and method, help students establish English learning mode, ensure the high marks high talent training philosophy at the same time with improve English learning interest.

\section{References}

[1] Luo Yana. Discussion on English Vocabulary Teaching from the Angle of Metaphor in Cognitive linguistics. Teaching and Management (Theoretic version),2014,22(01):112-114.

[2] Ma Hao. Discussion on the Application of Cognitive Linguistics in the College English Vocabulary Teaching. Course Education Research (New teacher's teaching),2015,39(12):236-236.

[3] Yuan Honghe, Wu Keyan. English Vocabulary Research under the Cognitive Linguistics Theory. Journal of Hubei Economy Academy (Humanity and Social Science Edition), 2015,42(07):211-212.

[4] Li Ling, Wang Yaofen. Several Enlightenment of Cognitive Linguistics Theory on English Vocabulary Teaching. Crazy English (Teacher edition),2014,42(01):80-82.

[5] Zhang Fan. Teacher-student Interaction of English Vocabulary Class in the Middle School under the Cognitive Linguistics Theory. Journal of Suzhou Institute of Education, 2014,17(01):112-113,120. 